\title{
Non-innocent hybrid cycloolefin ligands for palladium/olefin cooperative catalysis
}

\author{
Ya-Xin Zheng $^{1}$ and Lei Jiao ${ }^{1 *}$
}

\author{
Affiliations: \\ ${ }^{1}$ Center of Basic Molecular Science (CBMS), Department of Chemistry, Tsinghua \\ University; Beijing, 100871, China. \\ *Corresponding author. Email: leijiao@mail.tsinghua.edu.cn
}

\begin{abstract}
Catalyst design is a key research area in modern synthetic chemistry. Engineering of molecular catalysts in homogeneous catalysis brings about new catalytic modes that enable efficient synthetic transformations. In this regard, design of novel ligands for transition-metal catalysis have played a major role. Olefins have been emerging as a significant class of steering ligands in transition-metal catalysis, which are known to serve as innocent ligands that provide electronic and steric tuning of the metal center. However, it is unknown whether a distinct type of olefin ligand that contrasts the common innocent feature is possible. Here we show that a novel type of heteroatom-cycloolefin hybrid ligand functions as a non-innocent ligand in palladium catalysis, which exhibits covalent catalytic function that enables efficient ipso,orthodifunctionalization of iodoarenes. Detailed mechanistic study revealed that this ligand undergoes reversible covalent bonding between the substrate and the cycloolefin unit, which forms key organopalladium intermediates to enable new reactivity. Our results demonstrate a novel design concept that utilizes unstrained cycloolefin as a covalent catalytic module, opening an avenue to a more general transition metal/olefin cooperative catalysis.
\end{abstract}




\section{Main:}

\section{Introduction}

Homogeneous transition-metal (TM) catalysis has made great contributions to modern synthetic chemistry ${ }^{1}$. Engineering of TM-based molecular catalysts played a crucial role, which enabled new catalytic modes that lead to useful synthetic reactions with enhanced selectivity and efficiency. In this regard, design of novel ligands for TM catalysis with specific functions is of great significance ${ }^{2}$.

The majority of ancillary ligands utilize one or more heteroatom (e.g., N, O, S, or P) as the coordination site to form the metal-ligand bond. In the past decades, olefins have been recognized as unique steering ligands in TM-catalysis ${ }^{3-7}$. Owing to elegant design, in these systems olefins do not behave as unsaturated reactants as usual, but act as spectator ligands to provide electronic and steric tuning of the metal center. A variety of diene and hybrid heteroatom/olefin ligands have been developed and used in combination with transition metals to promote many synthetically useful reactions (Fig. 1a). This great success led us to think of an interesting question contrasting the established concept of olefin ligand: is it possible to develop an olefin ligand that is reactive towards organometallic intermediates for transition metal catalysis, [i.e., a non-innocent (or cooperative) ligand ${ }^{8-11}$ ]? With this question in mind, we imagined to explore the possibility of developing a fundamentally new type of olefin ligand that makes use of the reactivity of the carbon-carbon double bond.
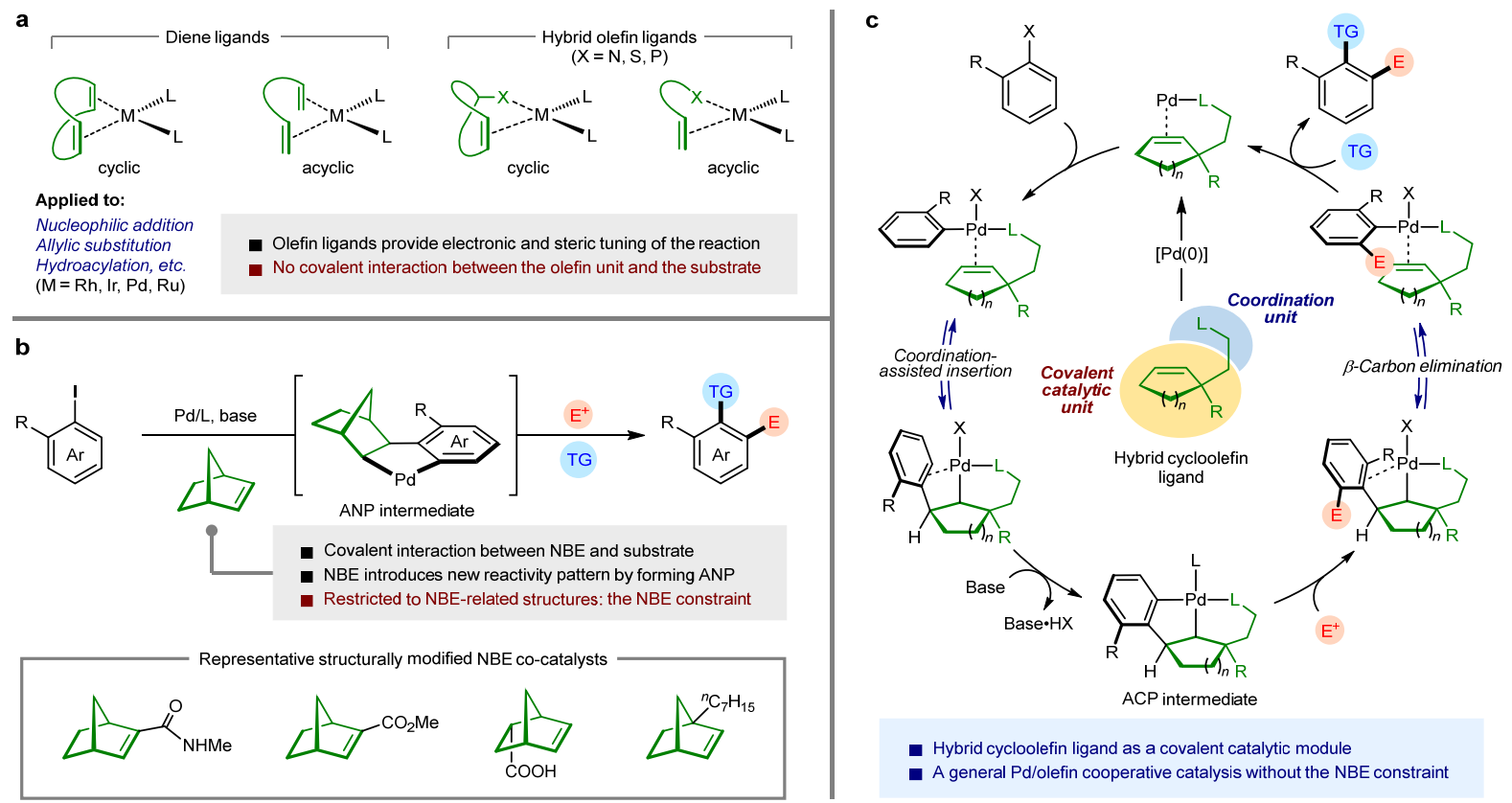

Fig. 1. Olefin ligands and co-catalysts. a, Olefins serve as stirring ligands to tune transitionmetal-catalyzed reactions electronically and sterically without covalent interaction with substrates. $\mathbf{b}$, In the Pd/NBE cooperative catalysis NBE serves as a covalent transient mediator to enable ipso,ortho-difunctionalization, and the bicycle[2.2.1] heptane skeleton is indispensable. c, In this work hybrid cycloolefin ligand has been developed as a covalent catalytic module, which enables a general Pd/olefin cooperative catalysis.

This idea was inspired by the palladium/norbornene (Pd/NBE) cooperative catalysis (Fig. $1 b)^{12-18}$, which was originally discovered by Catellani and co-workers ${ }^{19}$, and further 
implemented into a general synthetic methodology by the Lautens group ${ }^{20,21}$ and many other research groups. In this chemistry, NBE reacts with an arylpalladium species to form an arylnorborylpalladacycle (ANP) intermediate, which then allows for sequential introduction of an electrophile $\left(\mathrm{E}^{+}\right)$and a termination group (TG) at the ipso- and ortho-positions of the aryl ring, and finally regenerates NBE. On a catalytic viewpoint, NBE represents a unique olefintype covalent co-catalyst that bonds to the substrate, enables new reactivity, and recovers its original structure while releasing the product. While in most cases this co-catalyst should be used in sub- or even super-stoichiometric amounts to ensure reactivity, this process has been regarded as an efficient protocol for vicinal difunctionalization of arene substrates. However, the $\mathrm{Pd} / \mathrm{NBE}$ cooperative catalysis is constrained to $\mathrm{NBE}$ and its structurally modified derivatives in which the bicyclo[2.2.1] heptene skeleton remains ${ }^{22-28}$, and to date people have to admit that the NBE structure is indispensable due to its unique structural feature ${ }^{17}$. It has been a long-standing question that whether or not an unstrained olefin without the NBE skeleton could be used in the covalent cooperative catalysis ${ }^{29}$. This question, together with the question before, represent the quest for a new type of cooperative olefin ligand. We intended to address these questions, not only for sake of curiosity, but also for exploiting the potential of metal-olefin cooperative catalysis. Here, we report that hybrid cycloolefin ligands could function as a covalent catalytic module in combination with $\mathrm{Pd}$ to achieve a $\mathrm{Pd} /$ olefin cooperative catalysis, and a detailed mechanistic study clearly revealed the non-innocent nature of the ligand.

\section{Results and Discussion}

\section{Ligand Design}

We envisioned to design a molecule consisting of a cycloolefin moiety and a coordination site as the proposed hybrid cycloolefin ligand (Fig. 1c). In this ligand, the coordination site is tethered to the $\alpha$-position of the cycloolefin, aiming to facilitate alkene insertion into an arylpalladium species by coordination. This directed insertion could exclude the existence of a hydrogen atom syn to palladium, avoiding undesired $\beta$-hydride elimination. The cycloolefin framework keeps the aryl group and the palladium center suprafacial after the insertion step, enabling arylcycloalkylpalladacycle (ACP) formation under basic conditions. We hypothesized that, this ACP intermediate may behave similarly to the ANP intermediate ${ }^{12}$, allowing a reaction sequence involving the introduction of an electrophile at the ortho position, regeneration the cycloolefin motif by $\beta$-carbon elimination, and the attachment of a termination group at the ipso position.

The first ligand designed for this propose (L1) features a cyclopentenyl group as the cycloolefin unit, a diarylphosphine group as the coordination unit, and a flexible ethylene bridge uniting both units. To test its performance on cooperative catalysis, the orthoalkylation/ipso-Heck reaction ${ }^{19}$ of aryl iodide 1a with alkyl iodide $\mathbf{2 a}$ and acrylate 3a was chosen as the model reaction (Fig. 2a). To our excitement, the desired difunctionalization product 4a was obtained in a moderate yield, together with the Heck-type byproduct 5a. Not surprisingly, a phosphine oxide derivative of this ligand, L1-O, exhibited complete loss of activity for promoting the formation of $\mathbf{4 a}$, while the byproduct $\mathbf{5 a}$ was still produced. The success of ligand $\mathbf{L 1}$ was a proof-of-concept that showcased the competence of the phosphinecycloolefin hybrid ligand in $\mathrm{Pd} /$ olefin cooperative catalysis, though improvements on both activity and selectivity were still demanded. 

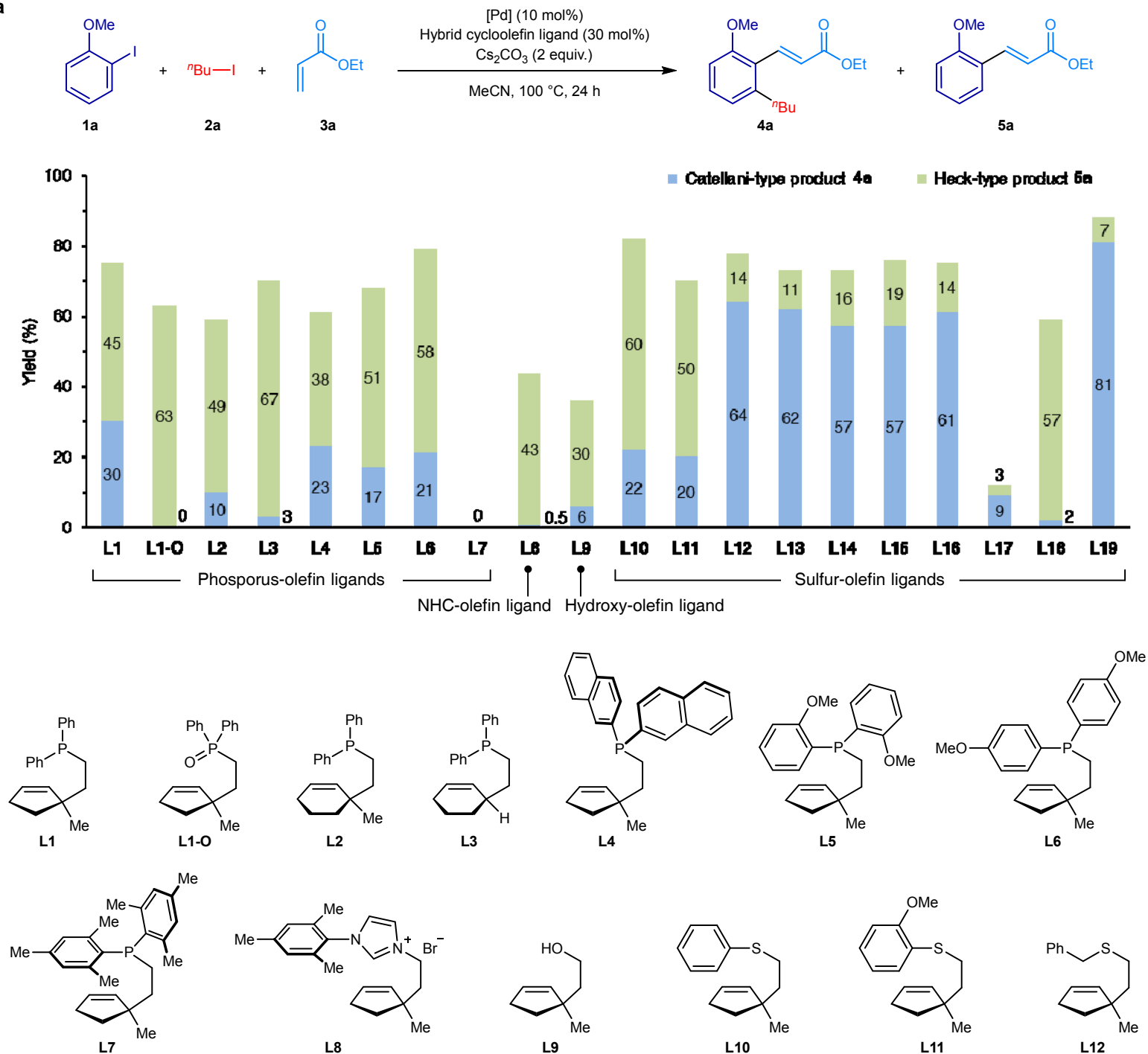

L8

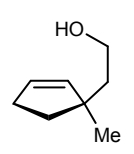

L9

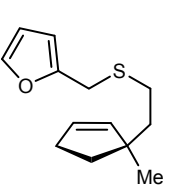

L16

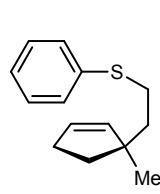

L10
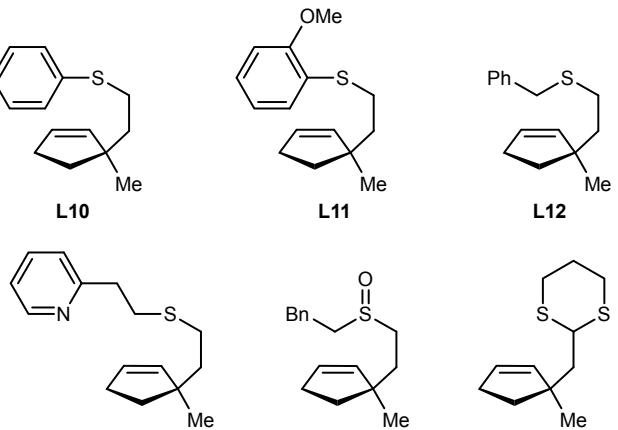

L17

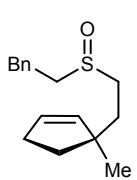

L18

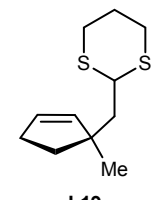

L19

b

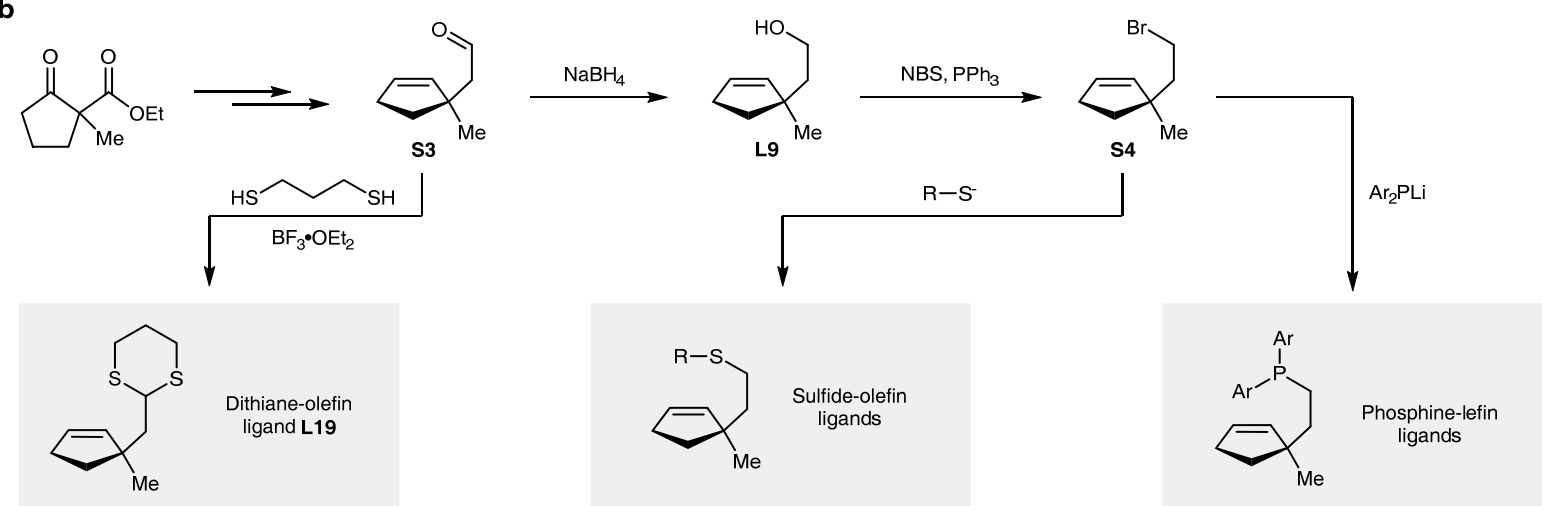

Fig. 2. Hybrid olefin ligands for Pd/olefin cooperative catalysis. a, Structure-activity relationship. Reaction conditions: iodoarene $1 \mathbf{a}(0.2 \mathrm{mmol})$, iodide $\mathbf{2 a}(0.4 \mathrm{mmol})$, acrylate $\mathbf{3 a}$ 
(0.4 mmol), the palladium source [0.02 mmol, for ligands L1-L8, Pd(OAc) 2 was employed; for ligands L9-L19, $\mathrm{Pd}(\mathrm{dba})_{2}$ was employed ( $\mathrm{dba}=$ dibenzylideneacetone)], ligand (0.06 mmol), $\mathrm{Cs}_{2} \mathrm{CO}_{3}$ (0.4 mmol for L1-L7, $0.8 \mathrm{mmol}$ for $\mathbf{L 8}$ and $\mathbf{L 9}, 0.6 \mathrm{mmol}$ for $\mathbf{L 1 0}-\mathbf{L 1 9}$ ), acetonitrile $(2 \mathrm{~mL})$ as the solvent, $100{ }^{\circ} \mathrm{C}$, sealed tube, $24 \mathrm{~h}$. b, Representative synthetic routes of phosphine-, sulfide-, and dithiane-olefin ligands.

\section{Ligand Structure-Activity Relationship (SAR)}

We then focused on improving the performance of the hybrid cycloolefin ligand (Fig. 2a). Switching the cycloolefin ring system from cyclopentene to cyclohexene (L2) resulted a decreased reactivity. The $\alpha$-methyl group on the cycloolefin seemed to be beneficial, as removal of it (L3) led to significantly decreased activity in the cooperative catalysis. We then turned back to the cyclopentenyl unit and investigated the effect of the aryl substituents of the phosphine unit. However, to our disappointment, varying the aryl group between naphtha-2-yl (L4), 2-methoxyphenyl (L5), 4-methoxyphenyl (L6), and 2,4,6-mestyl (L7) groups did not improve the performance of the ligand. Given that the nature of the coordination site may dramatically alter the reactivity of the metal center, we attempted to use an $N$-heterocyclic carbene (NHC) motif (L8) or a hydroxyl group (L9) as the coordination unit in place of phosphine. However, both ligands were found unsatisfactory, though tiny amounts of the desired product 4 were still produced.

Interestingly, sulfide ligands, which are not prevalently used in palladium catalysis ${ }^{30-33}$, were found to be superior. Aryl sulfide ligands L10 and L11 exhibited comparable activity to that of phosphine ligands, while alkyl sulfide ligands L12-16 performed much better. It was found that, the substituent on the alkyl group exerts a minor effect on ligand activity, except that a coordinating pyrid-2-yl group (L17) suppresses the activity remarkably. A sulfoxidecycloolefin ligand L18 (as a 1:1 diastereomeric mixture) also exhibited a low reactivity. Quite interestingly, dithioacetal-cyclopentene ligand $\mathbf{L 1 9}$ exhibited the best activity amongst all ligands tested, which minimized the formation of the Heck product $\mathbf{5 a}$ and afforded the difunctionalization product $\mathbf{4 a}$ in an excellent yield. Overall, the SAR study identified hybrid cycloolefin ligand as a generic design for Pd/olefin cooperative catalysis, and revealed the performance of the hybrid cycloolefin ligand as a function of the coordination site. Most of these ligands are easily accessible from common precursors (Fig. 2b).

A series of control experiments were performed with the optimal ligand L19 to understand the role of each reaction component (Table 1). Not surprisingly, it was found that both palladium and the hybrid cycloolefin ligand $\mathbf{L 1 9}$ were indispensable for the catalysis (entries 1-3). $\mathrm{Pd}(\mathrm{dba})_{2}$ as the source of palladium instead of $\mathrm{PdCl}_{2}(\mathrm{MeCN})_{2}$ exerted a minor influence on the efficiency of the reaction (entry 4). Potassium carbonate was found to be incompetent as the base compared with cesium carbonate (entry 5). $N, N$-dimethylformamide (DMF) is also a compatible solvent, albeit somewhat inferior to acetonitrile (entry 6). Performing the reaction at $80{ }^{\circ} \mathrm{C}$ resulted in a decreased yield (entry 7). Finally, we were delighted to find that only 5 mol\% Pd and $10 \mathrm{~mol} \%$ of $\mathbf{L 1 9}$ were sufficient to promote the desired difunctionalization reaction efficiently (entry 8). Lowering the loading of $\mathbf{L 1 9}$ from $10 \mathrm{~mol} \%$ to $6 \mathrm{~mol} \%$ still resulted in satisfactory activity of the catalytic system, albeit minor decrease in yield and selectivity was observed (entries 9 and 10). For comparison, the reaction using $30 \mathrm{~mol} \% \mathrm{NBE}$ instead of L19 was far less efficient (entry 11), demonstrating the advantage of the new ligand in $\mathrm{Pd} /$ olefin cooperative catalysis. 
Table 1. Control experiments ${ }^{a}$

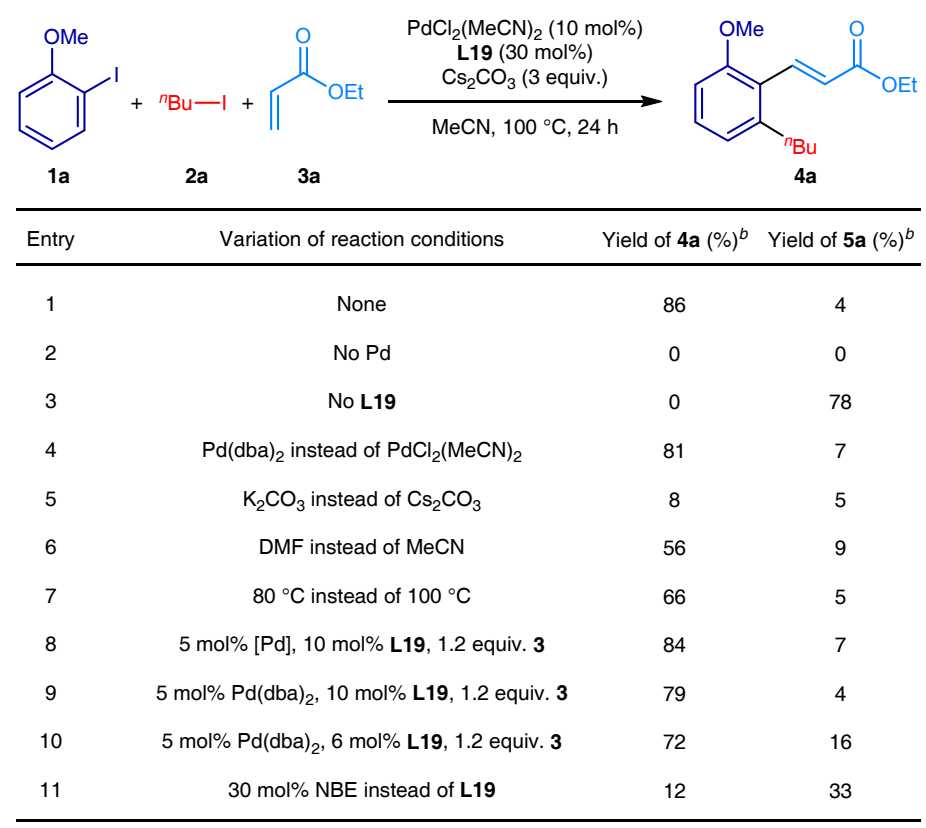

${ }^{a}$ Reaction conditions: iodoarene $\mathbf{1 a}(0.2 \mathrm{mmol})$, alkyl iodide $\mathbf{2 a}(0.4 \mathrm{mmol})$, acrylate $\mathbf{3 a}(0.4$ $\mathrm{mmol}), \mathrm{PdCl}_{2}(\mathrm{MeCN})_{2}(0.02 \mathrm{mmol}), \mathbf{L 1 9}(0.06 \mathrm{mmol}), \mathrm{Cs}_{2} \mathrm{CO}_{3}(0.6 \mathrm{mmol})$, acetonitrile (2 $\mathrm{mL}$ ) as the solvent, $100{ }^{\circ} \mathrm{C}$, sealed tube, 24 h. ${ }^{b}$ Yields were determined by ${ }^{1} \mathrm{H}$ NMR using 1,3,5-trimethoxybenzene as the internal standard.

\section{Substrate Scope}

With the optimal reaction conditions in hand, the substrate scope of this $\mathrm{Pd} /$ olefin cooperative catalysis was explored (Table 2). In the ortho-alkylation/ipso-Heck reaction, a series of iodoarene substrates with different 2-substituents, including methoxy (4a), methyl (4b), isopropyl (4c), trifluoromethyl (4d), carboxylic ester (4e), phenyl (4f), and chloro (4g), were found to be compatible. 2,4-Disubstituted iodobenzene $\mathbf{4 h}$ and $\alpha$-iodonaphthalene (4i) were also suitable substrates, affording the difunctionalization products in excellent yields. When iodoarenes without an ortho substituent were employed, both ortho positions were alkylated in good yields $(\mathbf{4 j}-\mathbf{m})$, and the selective mono ortho-alkylation was not achieved even if only one equivalent of the alkyl electrophile was employed.

The scope of the alkyl electrophile was also tested in the ortho-alkylation/ipso-Heck reaction. It was found that, primary alkyl iodides bearing a variety of functional groups served as suitable electrophiles for the cooperative catalysis (4n-u), while a tertiary carbon center at the $\beta$-position slightly diminished the reactivity $(\mathbf{4 v})$. It was noteworthy that, methyl triflate (MeOTf) was a perfect electrophile ${ }^{34}$ that allowed for efficient ortho-methylation/ipso-Heck reaction (4q), while $n$-butyl bromide exhibited decreased reactivity compared with $n$-butyl iodide (4a). 
Table 2. ipso,ortho-Difunctionalization of iodoarenes catalyzed by $\mathrm{Pd} / \mathbf{L 1 9}^{a}$

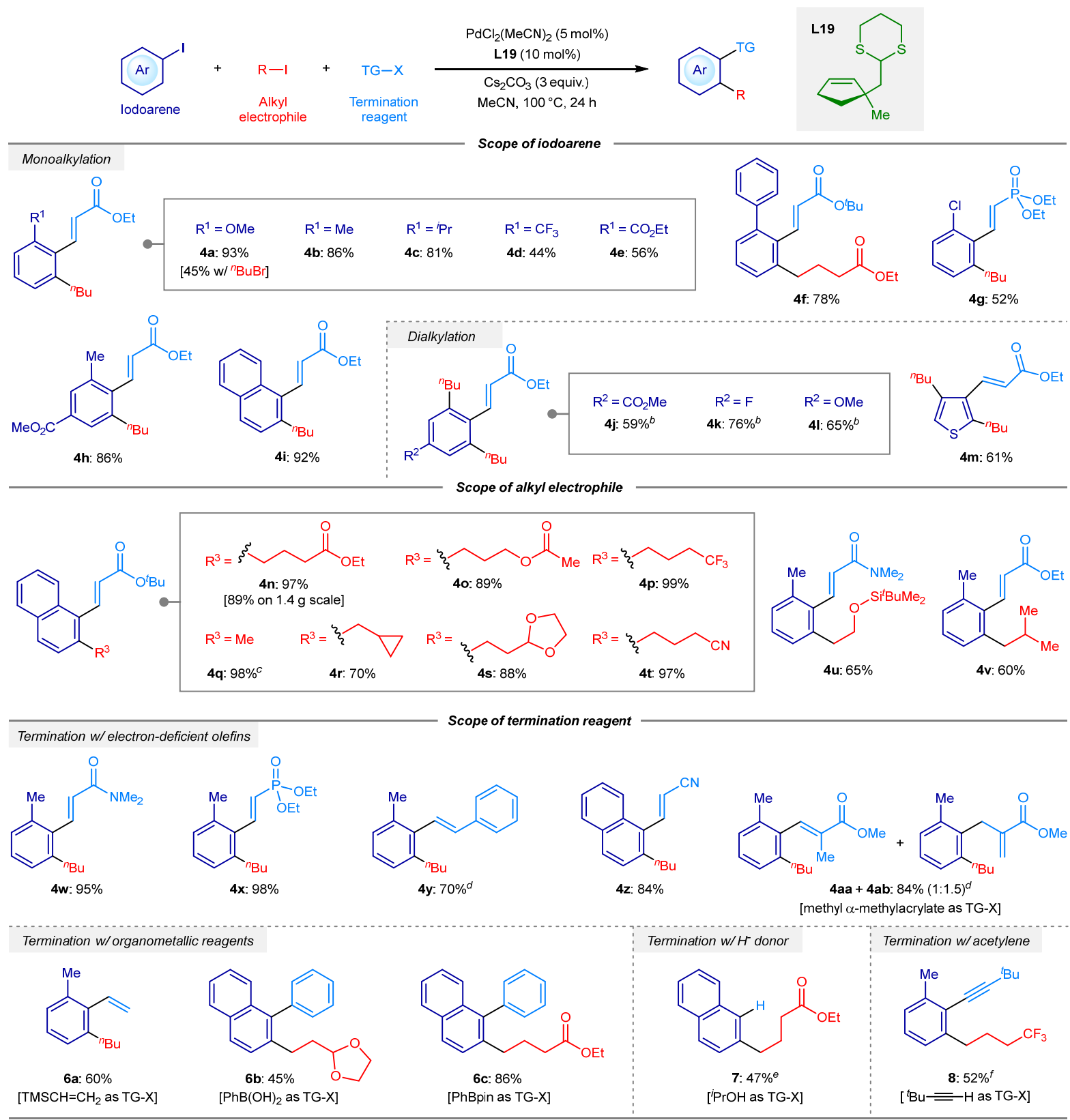

${ }^{a}$ Reaction conditions: iodoarene $1(0.4 \mathrm{mmol})$, alkyl iodide $2(0.8 \mathrm{mmol})$, termination reagent $3(0.48 \mathrm{mmol}), \mathrm{PdCl}_{2}(\mathrm{MeCN})_{2}(0.02 \mathrm{mmol}), \mathbf{L 1 9}(0.04 \mathrm{mmol}), \mathrm{Cs}_{2} \mathrm{CO}_{3}(1.2 \mathrm{mmol})$, acetonitrile $(4 \mathrm{~mL})$ as the solvent, $100{ }^{\circ} \mathrm{C}$, sealed tube, $24 \mathrm{~h}$. Yields of isolated products were reported. ${ }^{b} 1.2$ mmol (3 equiv.) of the alkyl iodide and $1.6 \mathrm{mmol}$ (4 equiv.) of $\mathrm{Cs}_{2} \mathrm{CO}_{3}$ was used. ${ }^{c} \alpha$ Iodonaphthalene $(0.8 \mathrm{mmol})$, methyl triflate $(0.8 \mathrm{mmol})$, tert-butyl acrylate $(0.4 \mathrm{mmol})$, $\mathrm{Pd}(\mathrm{dba})_{2}(0.04 \mathrm{mmol}), \mathbf{L 1 9}(0.06 \mathrm{mmol})$, and $\mathrm{Cs}_{2} \mathrm{CO}_{3}(1.0 \mathrm{mmol})$ were used. ${ }^{d} 1.0 \mathrm{mmol}$ (2.5 equiv.) of the electron-deficient olefin was used. ${ }^{e} 0.2 \mathrm{mmol}$ ( 0.5 equiv.) of isopropanol was used as the termination reagent. ${ }^{f} 1.2 \mathrm{mmol}$ ( 3 equiv.) of the alkyl iodide was used.

A variety of termination pathways were found viable in this catalytic system. Electrondeficient alkenes, including acrylamide $(\mathbf{4 w})$, vinylphosphonate $(\mathbf{4 g}$ and $\mathbf{4 x})$, styrene $(\mathbf{4 y})$, acrynitrile $(\mathbf{4 z})$, and $\alpha$-methylacrylate $(\mathbf{4 a a}+\mathbf{4 a b})$, served as appropriate termination reagents 
to afford the products in good to excellent yields. Organometallic reagents, including vinylsilane $(\mathbf{6 a})^{35}$, phenylbronic acid $(\mathbf{6 b})^{36}$, and phenylbronic ester $(\mathbf{6 c})^{37}$, were employed to produce the ortho-alkylation/ipso-coupling products in satisfactory yields. The use of isopropanol as a hydride donor ${ }^{38}$ resulted in the ortho-alkylation/ipso-hydrogenation product 7 in a moderate yield. Terminal alkyne as the termination reagent led to a decent yield of the ortho-alkylation/ipso-Sonogoshira coupling product $\mathbf{8}^{39}$.

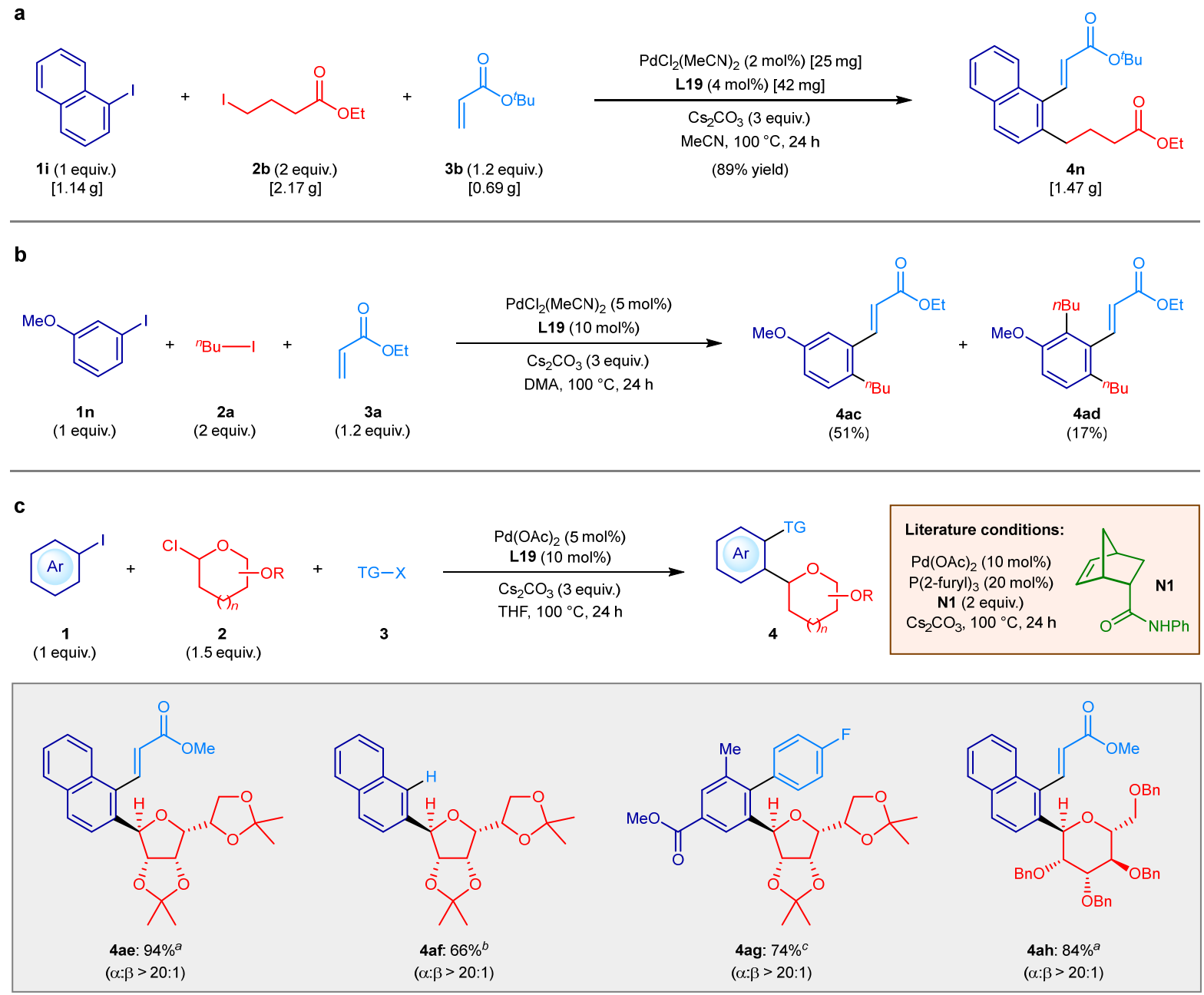

Fig. 3. Synthetic utility of the Pd/L19 catalytic system. a, Gram-scale synthesis of product 4n with a lower catalyst loading. b, Mono ortho-alkylation/ipso-Heck reaction of a metasubstituted iodoarene. c, Application in the ipso-functionalization-ortho-glycosylation reactions of aryl iodides. ${ }^{a} 1.2$ equiv. of methyl acrylate was used as the terminating reagent. ${ }^{b} 2$ equiv. of isopropanol was used as the terminating reagent. ${ }^{c} 2$ equiv. of 4-fluorophenylbronic acid pinacol ester was used as the terminating reagent and toluene was used as the solvent.

This highly efficient catalytic system enabled some synthetic tasks challenging for $\mathrm{Pd} / \mathrm{NBE}$ cooperative catalysis. First, difunctionalization of iodoarene $\mathbf{1 i}$ at a gram-scale could be performed with only $2 \mathrm{~mol} \%$ of Pd and $4 \mathrm{~mol} \%$ of $\mathbf{L 1 9}$ to afford a good yield of product $\mathbf{4 n}$, which was not possible in the $\mathrm{Pd} / \mathrm{NBE}$ cooperative catalysis with such low catalyst loading (Fig. 3a) ${ }^{17}$. Second, for an ortho-unsubstituted iodoarene (e.g., meta-substituted iodoarene), the $\mathrm{Pd} / \mathrm{NBE}$ catalytic system usually faces an "ortho-constraint" and fails to produce the 
difunctionalization product with selective installation of one ortho group. The Dong group developed a set of structurally-modified NBE catalysts to tackle this problem and achieved selective mono ortho-amination, -acylation and -arylation, while selective mono orthoalkylation remained underexplored ${ }^{40}$. Interestingly, we found that the $\mathrm{Pd} / \mathbf{L} \mathbf{1 9}$ system could promote mono ortho-butylation/ipso-Heck reaction of meta-substituted iodobenzene 1 n to produce the product 4ac in a reasonable yield, showcasing the potential of the designed olefin ligand to address the "ortho-constraint" in alkylative difunctionalization (Fig. 3b). Third, glycosyl chloride was recently found to be a new type of alkyl electrophile for the $\mathrm{Pd} / \mathrm{NBE}$ cooperative catalysis, but a superstoichiometric of structurally-modified NBE together with a phosphine ligand were found essential for catalytic activity ${ }^{41}$. Gratifyingly, the present $\mathrm{Pd} / \mathbf{L 1 9}$ catalytic system was able to promote the ortho-glycosylative difunctionalization of iodoarenes in a highly efficient and selective manner without using any additional ligand, and the yields were even superior as compared with the previous method (Fig. 3c).

Overall, the above experimental findings identify the dithiane-cyclopentene ligand L19 as a unique and versatile molecule, and its performance in $\mathrm{Pd} /$ olefin cooperative catalysis is even superior to that of NBE given that it lacks a highly strained ring system. This ligand exhibits perfect activity for promoting ortho-alkylation, while attempts to achieve other orthofunctionalization variants (e.g., amination and acylation) ${ }^{42,43}$ were unsuccessful at this stage. We hypothesized that this is probably due to the lack of a second vacant coordinate site on the ACP intermediate as a result of side-arm coordination.

\section{Reaction Mechanism}

The most significant aspect regarding the present cooperative catalysis is the reaction mechanism, in which the evidence for covalent catalytic behavior of the cycloolefin ligand is the key point. To our disappointment, attempts to isolate possible intermediates in the reaction catalyzed by $\mathrm{Pd} / \mathbf{L} 19$ were fruitless, which was probably due to their lability. Then we focused again on the less reactive phosphine-cycloolefin ligands (Fig. 4a). Gratifyingly, when iodoarene substrate 1a, $\mathrm{Pd}(\mathrm{II})$, and ligand $\mathbf{L} 4$ were heated in the presence of cesium carbonate, complex 9 was obtained in a reasonable yield. A more efficient procedure to prepare this complex employed trimethylamine to facilitate $\mathrm{Pd}(\mathrm{II})$ reduction, which avoided the use of excess phosphine-cycloolefin ligand $\mathbf{L 1 9}$ and remarkably increased the yield of complex 9. Single crystal X-ray diffraction (XRD) analysis unambiguously confirmed the structure of this complex, in which the ligand cyclopentyl unit bonds to both the substrate arene ring and the palladium center. The phosphine unit on the side-arm coordinates to Pd, which evidences the directed insertion of the cycloolefin moiety into the aryl-palladium bond.

With this complex in hand, the organometallic transformations in the cooperative catalysis were studied in a greater detail. Treatment of complex 9 with sodium methoxide or silver carbonate at room temperature led to ortho- $\mathrm{C}-\mathrm{H}$ palladation, which resulted in the clean formation of the ACP complex 10 (Fig. 4a). Nuclear magnetic resonance (NMR) analysis indicated that three neighboring protons remain on the methoxy-substituted aryl ring in complex 10, two of which exhibit descending ${ }^{31} \mathrm{P}-{ }^{1} \mathrm{H}$ coupling constants $\left(J_{\mathrm{P}-\mathrm{H}}\right)$. This is in agreement with the proposed palladacycle structure, in which the palladium forms a covalent bond with the aryl ring at the ortho-position. The identity of this complex was also supported by deuterium labeling experiments, in which the in-situ generated complex 10 was treated with excess deuterated acetic acid $\left(\mathrm{CH}_{3} \mathrm{COOD}\right)$ in methanol- $d_{4}\left(\mathrm{CD}_{3} \mathrm{OD}\right)$ to afford complex 9- $d$ with 
$85 \%$ ortho-deuterium incorporation on the aryl ring (Fig. 4b). Currently we are not aware of the identity of the ligand L binding to the vacant site of the ACP complex, which is probably a solvent molecule (acetonitrile).

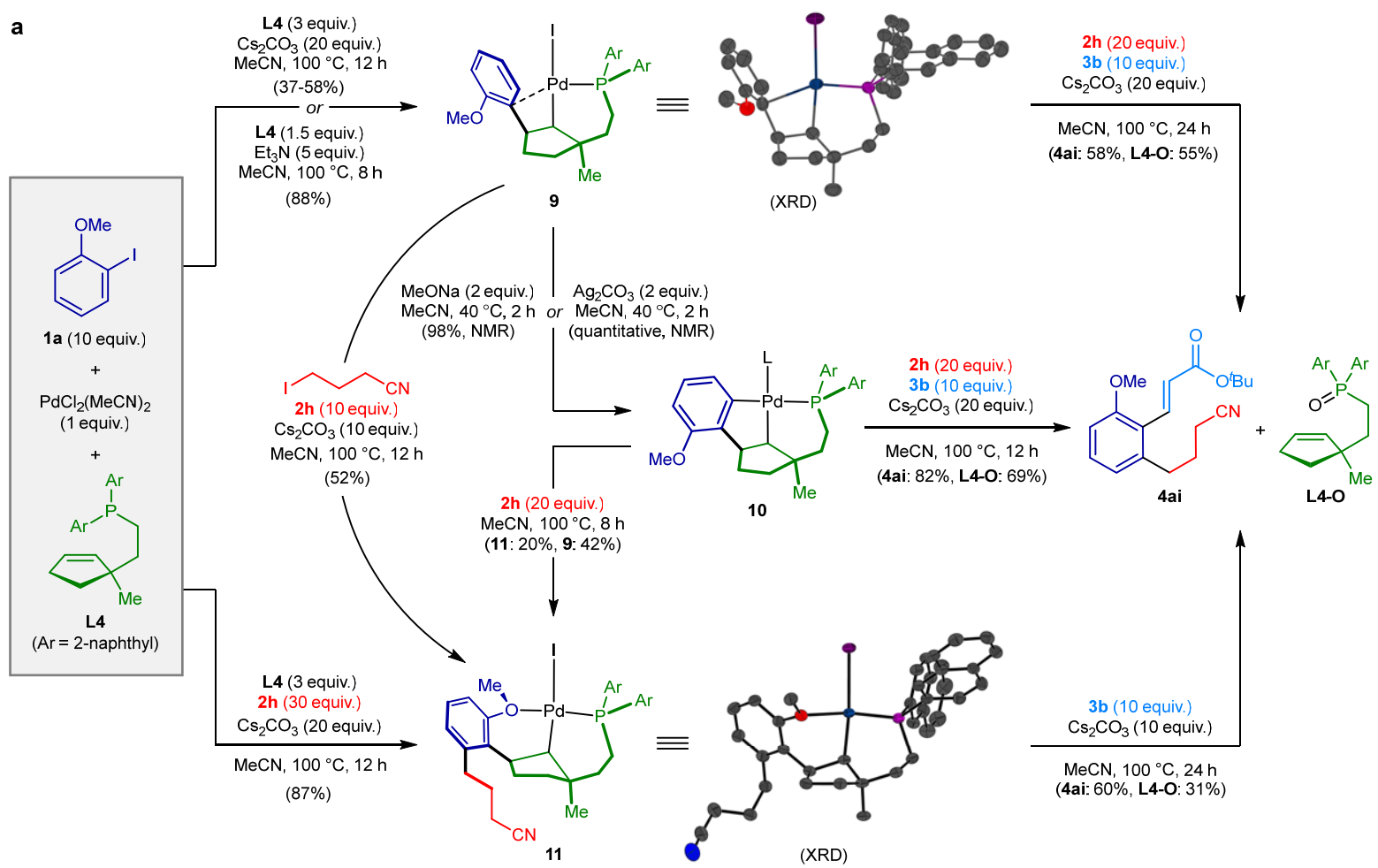

b
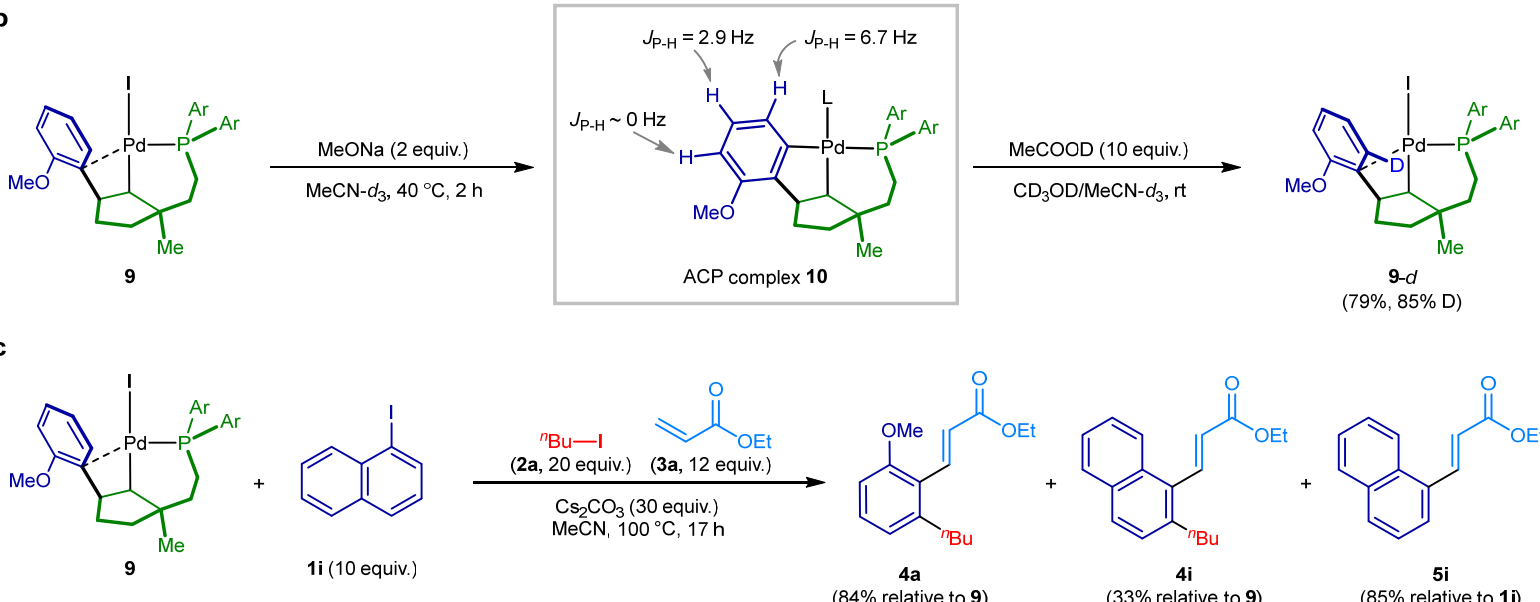

Fig. 4. Mechanism of the Pd/olefin cooperative catalysis with L4. a, The transformation network involving complexes $\mathbf{9}, \mathbf{1 0}$, and $\mathbf{1 1}$ demonstrated the non-innocent nature of the hybrid cycloolefin ligand. b, NMR analysis of ACP complex 10, together with the deuterium labeling experiment with deuterated acetic acid, verified the identity of this palladacycle intermediate. c, Crossover experiment employing complex $\mathbf{9}$ and aryl iodide $\mathbf{1 i}$ afforded two difunctionalizaiton products, which confirmed the regeneration of catalytically active species in the $\mathrm{Pd} /$ olefin cooperative catalysis after product release. 
The activity of ACP complex 10 towards alkyl electrophile was then studied (Fig. 4a). After heating a solution of $\mathbf{1 0}$ (prepared by deprotonation of complex 9 by $\mathrm{Ag}_{2} \mathrm{CO}_{3}$ ) and alkyl iodide $\mathbf{2 h}$ at $100{ }^{\circ} \mathrm{C}$, a new aryl-Pd-ligand complex 11, in which the ortho-position of the aryl ring was alkylated, was produced in $20 \%$ yield. XRD analysis confirmed its structure and disclosed a coordination mode different from complex 9, in which the oxygen atom in the ortho-methoxy group coordinates to $\mathrm{Pd}$ instead of the ipso-carbon, probably due to the steric bulkiness introduced after ortho-alkylation. It was found that, the ortho-alkylated complex 11 could be synthesized more efficiently under synthetically relevant conditions. By reacting complex 9 with excess alkyl iodide $2 \mathbf{h}$ and $\mathrm{Cs}_{2} \mathrm{CO}_{3}$, or by directly running the reaction between iodoarene 1a, $\mathrm{Pd}(\mathrm{II})$, and $\mathbf{L 2}$ in the presence of $\mathrm{Cs}_{2} \mathrm{CO}_{3}$, complex 11 was obtained in good yields.

All three aryl-Pd-ligand-type complexes were found to converge at the final difunctionalization product 4ai (Fig. 4a). Complexes $\mathbf{9}$ and $\mathbf{1 0}$ afforded product 4ai after treatment with excess $\mathbf{2 a}$ and $\mathbf{3 b}$ in the presence of $\mathrm{Cs}_{2} \mathrm{CO}_{3}$. When complex 11 was treated with excess acrylate $\mathbf{3 b}$, product $\mathbf{4 a i}$ was also produced in a reasonable yield. In particular, in all reactions the oxidized ligand L4-O was isolated in significant amounts, which is the evidence for the retro-insertion step in the cooperative catalysis.

The final step that closes the catalytic cycle of the Pd/olefin cooperative catalysis was supported by a crossover experiment (Fig. 4c). When complex 9 was reacted under synthetically relevant conditions with iodoarene $\mathbf{1} \mathbf{i}$, alkyl iodide $\mathbf{2 a}$, and acrylate $\mathbf{3 a}$, the formation of both difunctionalization products $\mathbf{4 a}$ and $\mathbf{4 i}$ was observed in addition to the Hecktype product 5i. Despite a low yield of the cross-product 4i, its formation definitely demonstrates that the alkylated organopalladium complex was able to release the final product while regenerating the reactive species for the next catalytic cycle. The above results clearly illustrate a transformation network for the Pd/olefin cooperative catalysis (Fig. 1c), in which the cycloolefin unit actively participates in the bond-forming and bond-breaking events.

\section{Conclusion}

In summary, in this work we show that hybrid cycloolefin ligand functions as a novel noninnocent ligand in palladium catalysis, which enables efficient ipso,ortho-difunctionalization of iodoarenes. The mode of action of this ligand has been elucidated by detailed mechanistic study, which provided direct evidences for reversible covalent bonding between the substrate and the cycloolefin unit that leads to the key organopalladium intermediates. The present work demonstrates a new concept in olefin ligand design, and opens an avenue to a more general $\mathrm{Pd}$ /olefin cooperative catalysis without the NBE constraint. We anticipate this ligand design to be a starting point for the development of olefin-based non-innocent ligands, which may lead to the discovery of more unprecedented reaction modes and catalytic systems that advance synthetic chemistry.

\section{Data availability}

The data supporting the findings of this study are available within the paper and its Supplementary Information. Metrical parameters for the structure of complex $\mathbf{9}$ and $\mathbf{1 1}$ (see Supplementary Information) are available free of charge from the Cambridge Crystallographic Data Centre (https://www.ccdc.cam.ac.uk/) under reference numbers CCDC 2060007, CCDC 2060009, respectively. 


\section{References}

1. Tsuji, J. Transition Metal Reagents and Catalysts: Innovations in Organic Synthesis. (John Wiley, 2002).

2. Ligand Design in Metal Chemistry: Reactivity and Catalysis. (Wiley-VCH, 2016).

3. Glorius, F. Chiral olefin ligands-new "spectators" in asymmetric catalysis. Angew. Chem. Int. Ed. 43, 3364-3366 (2004).

4. Defieber, C., Grützmacher, H. \& Carreira, E. M. Chiral olefins as steering ligands in asymmetric catalysis. Angew. Chem. Int. Ed. 47, 4482-4502 (2008).

5. Feng, X. \& Du, H. Synthesis of chiral olefin ligands and their application in asymmetric catalysis. Asian J. Org. Chem. 1, 204-213 (2012).

6. Li, Y. \& $\mathrm{Xu}, \mathrm{M} . \mathrm{H}$. Simple sulfur-olefins as new promising chiral ligands for asymmetric catalysis. Chem. Commun. 50, 3771-3782 (2014).

7. Dong, H.-Q., Xu, M.-H., Feng, C.-G., Sun, X.-W. \& Lin, G.-Q. Recent applications of chiral $N$-tert-butanesulfinyl imines, chiral diene ligands and chiral sulfur-olefin ligands in asymmetric synthesis. Org. Chem. Front. 2, 73-89 (2015).

8. van der Vlugt, J. I. Cooperative catalysis with first-row late transition metals. Eur. J. Inorg. Chem. 363-375 (2011).

9. Lyaskovskyy, V. \& de Bruin, B. Redox non-innocent ligands: versatile new tools to control catalytic reactions. ACS Catal. 2, 270-279 (2012).

10. Trincado, M. \& Grutzmacher, H. in Cooperative Catalysis (ed R. Peters) 67-110 (Wiley-VCH, 2015).

11. Berben, L. A., de Bruin, B. \& Heyduk, A. F. Non-innocent ligands. Chem. Commun. 51, 1553-1554 (2015).

12. Catellani, M. Catalytic multistep reactions via palladacycles. Synlett, 0298-0313 (2003).

13. Catellani, M., Motti, E. \& Della Ca, N. Catalytic sequential reactions involving palladacycle-directed aryl coupling steps. Acc. Chem. Res. 41, 1512-1522 (2008).

14. Martins, A., Mariampillai, B. \& Lautens, M. Synthesis in the key of Catellani: norbornene-mediated ortho C-H functionalization. Top. Curr. Chem. 292, 1-33 (2010).

15. Della Ca, N., Fontana, M., Motti, E. \& Catellani, M. Pd/norbornene: a winning combination for selective aromatic functionalization via $\mathrm{C}-\mathrm{H}$ bond activation. Acc. Chem. Res. 49, 1389-1400 (2016).

16. Wegmann, M., Henkel, M. \& Bach, T. C-H alkylation reactions of indoles mediated by $\mathrm{Pd}(\mathrm{II})$ and norbornene: applications and recent developments. Org. Biomol. Chem. 16, 5376-5385 (2018).

17. Wang, J. \& Dong, G. Palladium/norbornene cooperative catalysis. Chem. Rev. 119, 7478-7528 (2019).

18. Gu, Z., Zhao, K. \& Ding, L. Development of new electrophiles in palladium/norbornene-catalyzed ortho-functionalization of aryl halides. Synlett 30, 129-140 (2018).

19. Catellani, M., Frignani, F. \& Rangoni, A. A complex catalytic cycle leading to a regioselective synthesis of $o, o^{\prime}$-disubstituted vinylarenes. Angew. Chem. Int. Ed. 36, 119-122 (1997).

20. Lautens, M. \& Piguel, S. A new route to fused aromatic compounds by using a palladium-catalyzed alkylation - alkenylation sequence. Angew. Chem. Int. Ed. 39, 1045-1046 (2000). 
21. Bressy, C., Alberico, D. \& Lautens, M. A route to annulated indoles via a palladiumcatalyzed tandem alkylation/direct arylation reaction. J. Am. Chem. Soc. 127, 1314813149 (2005).

22. Li, R. \& Dong, G. Structurally modified norbornenes: a key factor to modulate reaction selectivity in the palladium/norbornene cooperative catalysis. J. Am. Chem. Soc. 142, 17859-17875 (2020).

23. Dong, Z., Wang, J., Ren, Z. \& Dong, G. Ortho C-H acylation of aryl iodides by palladium/norbornene catalysis. Angew. Chem. Int. Ed. 54, 12664-12668 (2015).

24. Shen, P. X., Wang, X. C., Wang, P., Zhu, R. Y. \& Yu, J. Q. Ligand-enabled meta-C-H alkylation and arylation using a modified norbornene. J. Am. Chem. Soc. 137, 1157411577 (2015).

25. Liu, J., Ding, Q., Fang, W., Wu, W., Zhang, Y. \& Peng, Y. Pd(II)/norbornene-catalyzed meta-C-H alkylation of nosyl-protected phenylalanines. J. Org. Chem. 83, 1321113216 (2018).

26. Liu, Z.-S., Qian, G., Gao, Q., Wang, P., Cheng, H.-G., Wei, Q., Liu, Q. \& Zhou, Q. Palladium/norbornene cooperative catalysis to access tetrahydronaphthalenes and indanes with a quaternary center. ACS Catal. 8, 4783-4788 (2018).

27. Cai, W. \& Gu, Z. Selective ortho thiolation enabled by tuning the ancillary ligand in palladium/norbornene catalysis. Org. Lett. 21, 3204-3209 (2019).

28. Ding, Y. N., Shi, W. Y., Liu, C., Zheng, N., Li, M., An, Y., Zhang, Z., Wang, C. T., Zhang, B. S. \& Liang, Y. M. Palladium-catalyzed ortho-C-H glycosylation/ipsoalkenylation of aryl iodides. J. Org. Chem. 85, 11280-11296 (2020).

29. Ye, J. \& Lautens, M. Palladium-catalysed norbornene-mediated C-H functionalization of arenes. Nat. Chem. 7, 863-870 (2015).

30. Mellah, M., Voituriez, A. \& Schulz, E. Chiral sulfur ligands for asymmetric catalysis. Chem. Rev. 107, 5133-5209 (2007).

31. Gorsline, B. J., Wang, L., Ren, P. \& Carrow, B. P. C-H alkenylation of heteroarenes: mechanism, rate, and selectivity changes enabled by thioether ligands. J. Am. Chem. Soc. 139, 9605-9614 (2017).

32. Naksomboon, K., Valderas, C., Gomez-Martinez, M., Alvarez-Casao, Y. \& FernandezIbanez, M. A. S,O-ligand-promoted palladium-catalyzed C-H functionalization reactions of nondirected arenes. ACS Catal. 7, 6342-6346 (2017).

33. Wang, L. \& Carrow, B. P. Oligothiophene synthesis by a general C-H activation mechanism: electrophilic concerted metalation-deprotonation (eCMD). ACS Catal. 9, 6821-6836 (2019).

34. Gao, Q., Shang, Y., Song, F., Ye, J., Liu, Z. S., Li, L., Cheng, H. G. \& Zhou, Q. Modular dual-tasked C-H methylation via the Catellani strategy. J. Am. Chem. Soc. 141, 1598615993 (2019).

35. Shi, G., Shao, C., Ma, X., Gu, Y. \& Zhang, Y. Pd(II)-catalyzed Catellani-type domino reaction utilizing arylboronic acids as substrates. ACS Catal. 8, 3775-3779 (2018).

36. Catellani, M., Motti, E. \& Minari, M. Symmetrical and unsymmetrical 2,6-dialkyl-1,1'biaryls by combined catalysis of aromatic alkylation via palladacycles and Suzuki-type coupling. Chem. Commun., 157-158 (2000).

37. Ye, C., Zhu, H. \& Chen, Z. Synthesis of biaryl tertiary amines through Pd/norbornene joint catalysis in a remote C-H amination/Suzuki coupling reaction. J. Org. Chem. 79, 
8900-8905 (2014).

38. Wilhelm, T. \& Lautens, M. Palladium-catalyzed alkylation-hydride reduction sequence: synthesis of meta-substituted arenes. Org. Lett. 7, 4053-4056 (2005).

39. Motti, E., Rossetti, M., Bocelli, G. \& Catellani, M. Palladium catalyzed multicomponent reactions in ordered sequence: new syntheses of $o, o^{\prime}-$ dialkylsubstituted diarylacetylenes and diarylalkylidenehexahydromethanofluorenes. $J$. Organomet. Chem. 689, 3741-3749 (2004).

40. Wang, J., Li, R., Dong, Z., Liu, P. \& Dong, G. Complementary site-selectivity in arene functionalization enabled by overcoming the ortho constraint in palladium/norbornene catalysis. Nat. Chem. 10, 866-872 (2018).

41. Lv, W., Chen, Y., Wen, S., Ba, D. \& Cheng, G. Modular and stereoselective synthesis of C-aryl glycosides via Catellani reaction. J. Am. Chem. Soc. 142, 14864-14870 (2020).

42. Dong, Z. \& Dong, G. Ortho vs ipso: site-selective Pd and norbornene-catalyzed arene C-H amination using aryl halides. J. Am. Chem. Soc. 135, 18350-18353 (2013).

43. Zhou, P.-X., Ye, Y.-Y., Liu, C., Zhao, L.-B., Hou, J.-Y., Chen, D.-Q., Tang, Q., Wang, A.-Q., Zhang, J.-Y., Huang, Q.-X., Xu, P.-F. \& Liang, Y.-M. Palladium-catalyzed acylation/alkenylation of aryl iodide: a domino approach based on the CatellaniLautens reaction. ACS Catal. 5, 4927-4931 (2015).

Acknowledgments: Financial support was provided by the National Natural Science Foundation of China (grant number 21822304). Prof. Ming-Tian Zhang is acknowledged for insightful discussions. The technological platform of CBMS is acknowledged for providing instrumentation.

Author contributions: L.J. and YX.Z. conceived the work and designed the experiments. YX.Z. performed the laboratory experiments (with help from L.J.). L.J. and YX.Z. analysed the data and co-wrote the manuscript.

Competing interests: Authors declare that they have no competing interests. 